\title{
OTIMIZAÇÃO ROBUSTA DE CARTEIRAS UTILIZANDO DESIGUALDADES MATRICIAIS LINEARES
}

\author{
Oswaldo L. V. Costa* \\ oswaldoelac.usp.br
}

\author{
Rodrigo B. Nabholz* \\ rodrigo.nabholz@poli.usp.br
}

* Departamento de Engenharia de Telecomunicações e Controle, Escola Politécnica da Universidade de São Paulo CEP: 05508-900, São Paulo, SP, Brasil

\begin{abstract}
The main goal of this paper is to formulate a robust meansemivariance portfolio selection problem in terms of a linear matrix inequalities (LMI) optimization problem. The meansemivariance model takes as the risk function a convex combination of the semivariances (below and above the expected return) of the tracking error (the difference between the investor's portfolio and a benchmark portfolio). We consider different forms of calculating the mean and semivariance of the tracking error. It is desired to minimize an objective function defined as a convex combination of the risk function minus the expected return of the tracking error. By a robust solution we mean a feasible portfolio which leads to a worst case value function lower than any other worst case value function evaluated at any other feasible portfolio. Numerical simulations will be presented with data from São Paulo Stock Exchange (BOVESPA).
\end{abstract}

KEYWORDS: Mean-semivariance, portfolio optimization, linear matrix inequalities, computational tool.

\section{RESUMO}

O objetivo deste trabalho é formular um problema de seleção robusta de ativos utilizando média-semivariância em termos de um problema de otimização com desigualdades matriciais lineares (LMI). O modelo de média-semivariância usa como função risco uma combinação convexa das semivariâncias

\footnotetext{
Artigo submetido em 09/12/02

1a. Revisão em 14/03/03; 2a. Revisão em 08/07/03

Aceito sob recomendação do Ed. Assoc. Prof. Takashi Yoneyama
}

(acima e abaixo do retorno esperado) do erro de rastreamento (a diferença entre os retornos da carteira considerada e um benchmark). Consideramos diferentes formas de calcular a média e a semivariância do erro de rastreamento. Iremos então minimizar uma função objetivo definida como uma combinação convexa da função risco menos o retorno esperado de erro de rastreamento. Chamamos de solução robusta a carteira factível que minimiza o valor da função objetivo no pior caso. Serão apresentadas simulações numéricas com dados de ações negociadas na Bolsa de Valores de São Paulo (BOVESPA).

PALAVRAS-CHAVE: Média-semivariância, desigualdades matriciais lineares, otimização de carteiras, ferramenta computacional.

\section{INTRODUÇÃO}

Neste trabalho iremos abordar modelos para a seleção ótima de ativos para a composição de uma carteira. Por ativos entende-se uma coleção de instrumentos financeiros nos quais um investidor pode aplicar o seu dinheiro (ex: ações, CDB's, poupança, etc.). Neste contexto, carteiras seriam um conjunto de ativos financeiros. O exemplo clássico de uma carteira é um fundo de investimento, onde o gestor aplica os recursos dos cotistas em ativos financeiros.

O modelo de média-variância para a seleção de ativos, introduzido por (Markowitz, 1959), formaliza um problema clássico em finanças, no qual deseja-se reduzir os riscos de uma carteira através da diversificação dos ativos. O principal objetivo é maximizar o retorno esperado para um dado nível de 
risco ou minimizar o risco esperado para um dado retorno esperado. A seleção ótima de ativos obtida através da resolução desses problemas é a ferramenta mais usada e conhecida para a alocação de capital (ver (Campbell et al., 1997), (Elton e Gruber, 1995), (Jorion, 1992)). Recentemente o modelo de média-variância foi estendido para incluir a otimização do erro de rastreamento (ver (Roll, 1992)) e modelos de semivariância (ver (Hanza e Janssen, 1998)). Neste caso, o gestor dos recursos é avaliado pela performance obtida em comparação com um benchmark pré-estabelecido. O problema da decisão de alocação neste caso é baseado na diferença entre o retorno obtido pelo gestor e o retorno do benchmark, o chamado erro de rastreamento ou "tracking error". No modelo de semivariância a função risco é uma combinação convexa das semivariâncias (acima e abaixo do retorno esperado) do erro de rastreamento. Como exemplos de trabalhos sobre o erro de rastreamento podemos mencionar (Roll, 1992) que considera o problema de minimizar a volatilidade do erro de rastreamento, e (Rudolf et al., 1999), que utiliza modelos lineares para minimização do erro de rastreamento. Outras funções objetivos para esta classe de problemas também podem ser consideradas (ver (Zenios, 1993)), e uma abordagem unificada destas metodologias pode ser encontrada em (Duarte Jr., 1999).

Como apontado em (Rustem et al., 1995), para a estratégia ótima do modelo de média-variância ser útil, os retornos esperados dos ativos e a matriz de covariância precisam ser estimativas precisas. Pequenas variações nos retornos podem provocar grandes mudanças na decisão de alocação, como pode ser visto em (Black e Litterman, 1991). Esta falta de robustez com relação à imprecisão inerente as estimativas dos retornos e variâncias motiva o estudo de modelos robustos de otimização. Neste caso, a otimização não é feita para um único caso de médias e variâncias, mas consideramos um conjunto de valores para estes parâmetros, que chamaremos de cenários. Estratégias robustas para carteira min-max são consideradas em (Rustem et al., 1995), onde deseja-se obter uma carteira que minimize o valor da função objetivo no pior caso (máximo sob os cenários). Apesar de ser uma solução de pior caso entre todos os cenários utilizados, na prática podemos obter uma boa estratégia, principalmente se analisarmos períodos de muita volatilidade e mercados emergentes. Em (Costa e Paiva, 2002) é proposta uma abordagem com desigualdades matriciais lineares para resolver numericamente o problema robusto de seleção ótima de carteiras. Assume-se que os retornos esperados dos ativos bem como a matriz de covariância pertencem a um politopo convexo definido por alguns vértices escolhidos pelo gestor. Dois problemas foram abordados: o primeiro foi encontrar a carteira de volatilidade mínima no pior caso do erro de rastreamento com uma performance esperada garantida mínima. O segundo foi obter a carteira com o maior retorno esperado no pior caso com uma máxima volatilidade do erro de rastreamento deter- minada. Mostrou-se que estes dois problemas são equivalentes a resolver problemas de otimização com desigualdades matriciais lineares. Outras referências sobre algoritmos robustos para finanças são (Howe e Rustem, 1997) e (Howe et al., 1996).

Devido ao grande número de técnicas computacionais rápidas e confiáveis disponíveis atualmente para problemas de otimização com desigualdades matriciais lineares (ver (Oliveira et al., 1994)), esta abordagem tem se mostrado uma ferramenta importante para a derivação de algoritmos numéricos em controle robusto e problemas relacionados. Em particular, algoritmos utilizando programação semi-definida para obtenção de controle para sistemas estocásticos foram apresentados recentemente (ver (Boyd et al., 1994), (Costa et al., 1997),(Geromel et al., 1991)). Indicamos estas referências para maiores detalhes sobre a implementação prática de tais algoritmos.

O principal objetivo deste artigo, baseado em (Costa e Nabholz, 2002), é utilizar desigualdades matriciais lineares para resolver um problema de otimização do erro de rastreamento robusto utilizando o critério de média-semivariância e considerando diferentes valores para os retornos esperados e semivariâncias. Vamos assumir que os retornos esperados dos ativos pertencem a um politopo convexo definido por alguns vértices escolhidos pelo gestor. Para o cálculo das semivariâncias, vamos considerar estimativas baseadas em dados históricos dos retornos dos ativos. Pesos decrescentes ao longo do tempo são aplicados ao conjunto de dados. Assumimos também que a matriz formada pelos pesos pertence a um politopo convexo definido por alguns vértices escolhidos pelo gestor. A idéia por trás desta hipotése é que estes parâmetros (expectativa de retornos e semivariâncias) não são exatamente conhecidos e dificilmente são estimados com precisão. A função objetivo que queremos minimizar é a combinação convexa da semivariância do erro de rastreamento menos o retorno esperado do erro de rastreamento. O problema de otimização robusta do erro de rastreamento neste caso é determinar a carteira que minimiza o pior caso (máximo considerando todas as possíveis combinações convexas dos parâmetros) da função objetivo. Acreditamos que esta técnica representa uma ferramenta computacional importante na direção de superar as principais limitações do modelo de otimização de média-variância mencionado acima, com foco especial para problemas reais.

Este artigo está organizado da seguinte maneira. A notação, resultados básicos e a formulação do problema que será considerado durante todo o trabalho são apresentados na seção 2. A seção 3 apresenta a formulação com desigualdades matriciais lineares para o problema de otimização de médiasemivariância para o caso do erro de rastreamento robusto. A Seção 4 apresenta simulações numéricas com ações da Bo- 
vespa. O artigo é concluído na seção 5 com os comentários finais. Todas as provas podem ser encontradas no apêndice.

\section{PRELIMINARES}

\subsection{Desigualdades Matriciais Lineares e Definições Básicas}

Vamos denotar por $\mathbb{R}$ e $\mathbb{R}^{n}$ o espaço dos reais e vetores $n$ dimensionais reais respectivamente. Para uma dada matriz $Q$, definimos $Q^{\prime}$ como a matriz transposta de $Q$ e $Q \geq 0$ ( $Q>0$ respectivamente) significa que a matriz simétrica $Q$ tem que ser semi-definida positiva (definida positiva). Para $x \in \mathbb{R}^{n}$ e $\mathbf{0}$ o vetor zero $n$-dimensional, a desigualdade $x \geq \mathbf{0}$ significa que cada elemento do vetor $x$ é maior ou igual a zero.

Vejamos alguns fatos básicos sobre desigualdades matriciais lineares, também conhecidas por LMI. Uma LMI (nãoestrita) tem a forma

$$
F(x)=F_{0}+\sum_{i=1}^{n} x_{i} F_{i} \geq 0
$$

sendo $x=\left(\begin{array}{c}x_{1} \\ \vdots \\ x_{n}\end{array}\right) \in \mathbb{R}^{n}$ as variáveis e as matrizes simétricas $F_{i}, i=0, \ldots, n$ dadas. Um resultado importante para converter desigualdades convexas não-lineares em uma formulação LMI é o chamado complemento de Schur, que apresentamos a seguir (ver (Saberi et al, 1995), pag 13).

Proposição 1 Suponha $R>0$. Então

$$
\left[\begin{array}{ll}
Q & S \\
S^{\prime} & R
\end{array}\right] \geq 0
$$

se e somente se

$$
Q-S R^{-1} S^{\prime} \geq 0
$$

Note que a primeira desigualdade na Proposição 1 está na forma de uma LMI, e a segunda está na forma de uma desigualdade convexa não-linear. Um problema de otimização com desigualdades matriciais lineares consiste em achar um $x$ factível (ou seja, achar $x$ tal que $F(x) \geq 0$ ) que minimize (ou maximize) uma função convexa $c(x)$. Como mostrado em (Boyd et al., 1994), (Ghaoui e Niculescu, 2000), existem atualmente algoritmos numéricos para resolução de problemas de otimização com desigualdades matriciais lineares bastante eficientes (por exemplo (Oliveira et al., 1994)), com o ótimo global encontrado em tempo polinomial ((Boyd et al., 1994), (Ghaoui e Niculescu, 2000)).

Um Corolário imediato da Proposição 1 é o seguinte:
Corolário 1 Suponha $Q \geq 0$ uma matriz $n$ por $n, x(i) \in$ $\mathbb{R}^{n}$, e $\sum_{i=1}^{k} \alpha(i)=1, \alpha(i) \geq 0, i=1, \ldots, k$. Então ${ }^{1}$

$$
\left\|Q^{\frac{1}{2}}\left(\sum_{i=1}^{k} \alpha(i) x(i)\right)\right\|^{2} \leq \sum_{i=1}^{k} \alpha(i)\left\|Q^{\frac{1}{2}} x(i)\right\|^{2} .
$$

Assim sendo vamos definir por conveniência, para $\mathbb{X}$ um espaço de vetores reais ou matrizes, e uma coleção de pontos $v(i) \in \mathbb{X}, i=1, \ldots, \kappa$, o politopo convexo $\operatorname{Con}\{v(1), \ldots, v(\kappa)\}$ como

$$
\begin{gathered}
\operatorname{Con}\{v(1), \ldots, v(\kappa)\}= \\
\left\{v \in \mathbb{X} ; v=\sum_{i=1}^{\kappa} \alpha(i) v(i), \sum_{i=1}^{\kappa} \alpha(i)=1, \alpha(i) \geq 0\right\} .
\end{gathered}
$$

A formulação acima será utilizada na caracterização da robustez no modelo para seleção de carteiras.

Para um vetor $x \in \mathbb{R}^{n}$, definimos os vetores $[x]_{+} \in \mathbb{R}^{n} \mathrm{e}$ $[x]_{-} \in \mathbb{R}^{n}$ da seguinte maneira

$$
[x]_{+}=\left(\begin{array}{c}
\max \left\{0, x_{1}\right\} \\
\vdots \\
\max \left\{0, x_{n}\right\}
\end{array}\right)
$$

$\mathrm{e}[x]_{-}=x-[x]_{+}$, sendo $x_{i}$ o $i$-ésimo componente do vetor $x$. Seja $\Lambda$ uma matriz diagonal $n$ por $n$ com componentes positivos. É fácil ver que

$$
[x]_{+}^{\prime} \Lambda[x]_{-}=0
$$

Desta forma definimos para $x \in \mathbb{R}^{n}, y \in \mathbb{R}^{n}, u \in \mathbb{R}^{n}$, $v \in \mathbb{R}^{n}$, e $\Lambda$ uma matriz diagonal $n$ por $n$ com elementos positivos,

$$
\begin{gathered}
\Upsilon(x, u)=\Lambda^{\frac{1}{2}}(u-x), \\
\Delta(y, v)=\Lambda^{\frac{1}{2}}(v+y), \\
g(x, y, u, v)=\theta\|\Upsilon(x, u)\|^{2}+(1-\theta)\|\Delta(y, v)\|^{2}
\end{gathered}
$$

$\operatorname{com} \theta \in[0,1]$. Suponha que queremos obter $u \in \mathbb{R}^{n}, v \in$ $\mathbb{R}^{n}, u \geq \mathbf{0}$ e $v \geq \mathbf{0}$ tal que minimize $g(x, y, u, v)$. Seja

$$
g(x, y)=\min _{u \geq \mathbf{0}, v \geq \mathbf{0}} g(x, y, u, v) .
$$

Proposição 2 Para quaisquer $u \geq \mathbf{0}, v \geq \mathbf{0}$,

$$
g(x, y, u, v) \geq \theta\left\|\Lambda^{\frac{1}{2}}[x]_{-}\right\|^{2}+(1-\theta)\left\|\Lambda^{\frac{1}{2}}[y]_{+}\right\|^{2}
$$

e o ponto de mínimo em (11) é atingido para $u=[x]_{+}, v=$ $-[y]_{-}$e $g(x, y)=\theta\left\|\Lambda^{\frac{1}{2}}[x]_{-}\right\|^{2}+(1-\theta)\left\|\Lambda^{\frac{1}{2}}[y]_{+}\right\|^{2}$.

\footnotetext{
${ }^{1}$ Vale ressaltar que a norma que estamos adotando ao longo de todo este trabalho é a norma Euclidiana, ou norma-2
} 


\subsection{Modelo para Seleção de Carteiras}

Vamos considerar um modelo financeiro no qual existam $N$ ativos com risco representados pelo vetor aleatório de retornos $A$ (tomando valores em $\mathbb{R}^{N}$ ), e um ativo livre de risco com retorno conhecido $r \in \mathbb{R}$. Por conveniência, iremos escrever $A(t)$ para denotar o vetor de retornos num determinado instante de tempo $t$ e por $A_{i}(t)$ a $i$-ésima componente deste vetor $A(t)$. O vetor aleatório de retornos $A$ tem vetor de médias $\mu \in \mathbb{R}^{N}$, e uma matriz de covariância $N \times N$ dimensional $\Omega$. Desta forma $A$ pode ser escrito como

$$
A=\mu+\epsilon
$$

com $\epsilon$ um vetor aleatório com média zero e matriz de covariância $\Omega$. É conveniente definir o vetor $\varphi \in \mathbb{R}^{N+1}$ como:

$$
\varphi=\left(\begin{array}{l}
\mu \\
r
\end{array}\right) \text {. }
$$

Uma carteira $\omega$ será dada por um vetor em $\mathbb{R}^{N}$ cujos componentes são os pesos investidos nos ativos com risco $A$, ou seja, a $i$-ésima componente $\omega_{i}$ de $\omega$ é a proporção da carteira investida no ativo $i$. Assumiremos que a carteira pertença então ao conjunto $\Gamma$, definido em termos da seguinte LMI:

$$
\Gamma=\left\{\omega \in \mathbb{R}^{N} ; F_{0}+\sum_{i=1}^{N} \omega_{i} F_{i} \geq 0\right\}
$$

onde $F_{i}, i=0, \ldots, N$ são matrizes simétricas dadas. $\mathrm{O}$ conjunto $\Gamma$ é conveniente para representar restrições do tipo: a soma das componentes da carteira ser menor ou igual a 1, e não permitir vendas a descoberto. Ou seja, restrições do tipo:

$$
\omega^{\prime} \mathbf{1} \leq 1,0 \leq \omega_{i}, i=1, \ldots, N
$$

nas quais 1 representa um vetor $N$-dimensional formado por 1 em todas posições. Neste caso, as matrizes $F_{i}$ tem dimensão $(N+1) \times(N+1)$. A matriz $F_{0}$ será formada por zeros exceto o elemento $(1,1)$, que será 1 . Para $i=1, \ldots, N$, as matrizes $F_{i}$ são

$$
F_{1}=\left[\begin{array}{cccc}
-1 & 0 & \ldots & 0 \\
0 & 1 & \ldots & 0 \\
\vdots & \vdots & \ddots & \vdots \\
0 & 0 & \ldots & 0
\end{array}\right], \ldots, F_{N}=\left[\begin{array}{cccc}
-1 & 0 & \ldots & 0 \\
0 & 0 & \ldots & 0 \\
\vdots & \vdots & \ddots & \vdots \\
0 & 0 & \ldots & 1
\end{array}\right]
$$

Consideremos agora que $\left(1-\omega^{\prime} \mathbf{1}\right)$ é investido num ativo livre de risco $r$. Assim sendo, o retorno do investidor é dado por

$$
\omega^{\prime} A+\left(1-\omega^{\prime} \mathbf{1}\right) r \text {. }
$$

Tomemos ainda uma carteira conhecida $\omega_{B}$ fornecida pelo gestor, chamada benchmark. O retorno do benchmark é como em (18), ou seja,

$$
\omega_{B}^{\prime} A+\left(1-\omega_{B}^{\prime} \mathbf{1}\right) r .
$$

De (18) e (19) temos que a diferença entre o retorno da carteira do investidor $\omega$ e o benchmark, definido como o erro de rastreamento $e(\omega)$, é

$$
e(\omega)=\left(\omega-\omega_{B}\right)^{\prime} A+\left(\omega_{B}-\omega\right)^{\prime} \mathbf{1} r .
$$

O valor esperado do erro de rastreamento $e(\omega)$, denotado por $\rho(\omega)$, segue das equações (13) e (20), e é dado por

$$
\begin{aligned}
\rho(\omega) & =\left(\omega-\omega_{B}\right)^{\prime} \mu+\left(\omega_{B}-\omega\right)^{\prime} \mathbf{1} r \\
& =\left(\omega-\omega_{B}\right)^{\prime}(\mu-\mathbf{1} r)
\end{aligned}
$$

e a variância (volatilidade), denotada por $\sigma^{2}(\omega)$, é

$$
\sigma^{2}(\omega)=\left(\omega-\omega_{B}\right)^{\prime} \Omega\left(\omega-\omega_{B}\right)
$$

Como mencionado anteriormente, estamos interessados em medir o risco através de uma combinação convexa das semivariâncias acima e abaixo da média do retorno do erro de rastreamento, que são definidas como segue: a semivariância abaixo da média é definida por

$$
\begin{aligned}
\eta^{-}(\omega) & =E\left((\min \{0, e(\omega)-\rho(\omega)\})^{2}\right) \\
& =E\left(\left(\min \left\{0,\left(\omega-\omega_{B}\right)^{\prime}(A-\mu)\right\}\right)^{2}\right)
\end{aligned}
$$

e a semivariância acima da média por

$$
\begin{aligned}
\eta^{+}(\omega) & =E\left((\max \{0, e(\omega)-\rho(\omega)\})^{2}\right) \\
& =E\left(\left(\max \left\{0,\left(\omega-\omega_{B}\right)^{\prime}(A-\mu)\right\}\right)^{2}\right)
\end{aligned}
$$

De (23) e (24), a função risco é definida, para $\theta \in[0,1]$, como

$$
\theta \eta^{-}(\omega)+(1-\theta) \eta^{+}(\omega) \text {. }
$$

Pode-se verificar que para $\theta=\frac{1}{2}$, temos $\frac{1}{2}\left(\eta^{-}(\omega)+\right.$ $\left.\eta^{+}(\omega)\right)=\frac{1}{2} \sigma^{2}(\omega)$. O parâmetro $\theta$ permite ao investidor ponderar os desvios de carteira em relação a sua média. Por exemplo, para $\theta=1$, a função risco seria a semivariância abaixo da média, penalizando apenas os retornos abaixo da média. Para maiores detalhes e propriedades desta função risco citamos (Hanza e Janssen, 1995), (Duarte Jr., 1999), (Markowitz, 1959).

Suponhamos que um conjunto de dados históricos $a_{i}(t)$, $t=1, \ldots T$, esteja disponível, e $a_{i}(t)$ representa o valor observado do retorno aleatório $A_{i}(t)$ no instante $t$. Vamos assim definir a matriz $\mathcal{A}$ como:

$$
\mathcal{A}=\left[\begin{array}{ccc}
a_{1}(1) & \ldots & a_{N}(1) \\
\vdots & \ddots & \vdots \\
a_{1}(T) & \ldots & a_{N}(T)
\end{array}\right]
$$


Portanto a $t$-ésima linha de $\mathcal{A}$ representa o valor observado de $A(t)$. Seja

$$
\Lambda=\left[\begin{array}{ccc}
\lambda(1) & \ldots & 0 \\
\vdots & \ddots & \vdots \\
0 & \ldots & \lambda(T)
\end{array}\right]
$$

onde $\lambda(t)$ representa o peso dado ao valor observado no instante $t$ quando estimamos os parâmetros $\eta^{-}(\omega), \eta^{+}(\omega)$. Assumiremos que $\sum_{t=1}^{T} \lambda(t)=1$. Das equações (23), (24), (26), uma estimativa para $\eta^{-}(\omega), \eta^{+}(\omega)$ usando $\mathcal{A}$, os pesos $\Lambda$, e a média $\mu$, denotadas por $\eta_{\mu, \Lambda}^{-}(\omega), \eta_{\mu, \Lambda}^{+}(\omega)$ respectivamente, seria dada por:

$$
\begin{gathered}
\eta_{\mu, \Lambda}^{-}(\omega)=\left\|\Lambda^{\frac{1}{2}}\left[\left(\mathcal{A}-\mathbf{1} \mu^{\prime}\right)\left(\omega-\omega_{B}\right)\right]_{-}\right\|^{2}= \\
=\sum_{t=1}^{T} \lambda(t)\left(\left[\sum_{i=1}^{N}\left(a_{i}(t)-\mu_{i}\right)\left(\omega_{i}-\omega_{i B}\right)\right]_{-}\right)^{2} \\
\eta_{\mu, \Lambda}^{+}(\omega)=\left\|\Lambda^{\frac{1}{2}}\left[\left(\mathcal{A}-\mathbf{1} \mu^{\prime}\right)\left(\omega-\omega_{B}\right)\right]_{+}\right\|^{2}= \\
=\sum_{t=1}^{T} \lambda(t)\left(\left[\sum_{i=1}^{N}\left(a_{i}(t)-\mu_{i}\right)\left(\omega_{i}-\omega_{i B}\right)\right]_{+}\right)^{2}
\end{gathered}
$$

\subsection{Seleção Robusta de Carteiras Utili- zando SemiVariância}

Vamos abordar nesta seção o problema de seleção robusta de carteiras utilizando semivariância como função risco, que é o objetivo principal deste trabalho. Vamos lembrar que se tivéssemos estimativas precisas para os parâmetros $\mu, r_{f}$ e $\eta_{\mu, \Lambda}^{-}(\omega), \eta_{\mu, \Lambda}^{+}(\omega)$ então o problema de otimização a ser resolvido seria, para $\delta \in[0,1], \theta \in[0,1]$, dado por:

$$
\inf _{\omega \in \Gamma} f_{\varphi, \Lambda}(\omega)
$$

com

$$
\begin{aligned}
f_{\varphi, \Lambda}(\omega)= & -(1-\delta)\left(\omega-\omega_{b}\right)^{\prime}\left(\mu-\mathbf{1} r_{f}\right) \\
& +\delta\left(\theta \eta_{\mu, \Lambda}^{-}(\omega)+(1-\theta) \eta_{\mu, \Lambda}^{+}(\omega)\right)
\end{aligned}
$$

(lembrando que $\left.\varphi=\left(\begin{array}{c}\mu \\ r_{f}\end{array}\right)\right)$. Vale ressaltar que $\delta$ representa o grau de aversão ao risco do investidor. À medida que $\delta$ aumenta de 0 para 1 , aumenta também a cautela do investidor. E podemos ver que, para $\delta=1$, o investidor deseja a carteira de mínimo risco, independente do retorno esperado, enquanto que para $\delta=0$, o interesse é na carteira de máximo retorno, desconsiderando seu risco.

Podemos ver, das equações (28) e (29), que as estimativas para $\eta^{-}(\omega), \eta^{+}(\omega)$ dependem diretamente de $\Lambda, \varphi$. Diferentes valores de $\Lambda, \varphi$ levam a diferentes valores da função objetivo (31). Consideremos assim a situação na qual desejase utilizar no problema de otimização (30) todos os possíveis valores dos parâmetros $\Lambda, \varphi$ dentro dos politopos convexos

$$
\Xi=\operatorname{Con}\{\varphi(1), \ldots, \varphi(\kappa)\},
$$

e

$$
\Sigma=\operatorname{Con}\{\Lambda(1), \ldots, \Lambda(\nu)\} .
$$

Vamos assumir novamente que $\sum_{t=1}^{T} \lambda(k, t)=1, k=$ $1, \ldots, \nu$, lembrando que $\lambda(k, t)$ são os elementos na diagonal de $\Lambda(k)$. É importante ressaltar que os valores $\varphi(1), \ldots, \varphi(\kappa)$ e $\Lambda(1), \ldots, \Lambda(\nu)$ são fornecidos pelo gestor da carteira, e portanto não estamos interessados neste estudo em maneiras de obtê-los. O parâmetro $\mu(i)$ irá representar diferentes estimativas para o retorno dos ativos de risco, seja em função de diversas formas de cálculo ou de diferentes cenários futuros do período no qual a carteira manter-se-á inalterada. De forma análoga os parâmetros $r_{f}(i)$ podem representar valores distintos que a taxa livre de risco pode assumir no mesmo período. Os parâmetros $\Lambda(k)$ denotam diferentes pesos que podemos considerar na estimativa da função risco nas equações (28), (29). Logo, a versão robusta do problema de otimização de carteiras apresentado em (30), (31) é dada por:

$$
\inf _{\omega \in \Gamma} \sup _{\varphi \in \Xi, \Lambda \in \Sigma} f_{\varphi, \Lambda}(\omega)
$$

Portanto estamos querendo determinar uma carteira $\omega^{*}$ tal que:

$$
\sup _{\varphi \in \Xi, \Lambda \in \Sigma} f_{\varphi, \Lambda}\left(\omega^{*}\right) \leq \sup _{\varphi \in \Xi, \Lambda \in \Sigma} f_{\varphi, \Lambda}(\omega) .
$$

para cada $\omega \in \Gamma$, ou seja, existem $\varphi \in \Xi, \Lambda \in \Sigma$ de forma que:

$$
\sup _{\varphi \in \Xi, \Lambda \in \Sigma} f_{\varphi, \Lambda}\left(\omega^{*}\right) \leq f_{\varphi, \Lambda}(\omega) .
$$

Note que é possível obtermos para algum $\bar{\varphi} \in \Xi, \bar{\Lambda} \in \Sigma$ :

$$
f_{\bar{\varphi}, \bar{\Lambda}}(\omega) \leq f_{\bar{\varphi}, \bar{\Lambda}}\left(\omega^{*}\right) \leq \sup _{\varphi \in \Xi, \Lambda \in \Sigma} f_{\varphi, \Lambda}\left(\omega^{*}\right) .
$$

Quanto maiores forem os conjuntos $\Xi$ e $\Sigma$, maiores as chances de encontrarmos $\bar{\varphi} \in \Xi, \bar{\Lambda} \in \Sigma$ satisfazendo (37). Logo a utilização de um número muito grande de cenários pode deteriorar a performance do método, dado que obteremos uma solução $\omega^{*}$ desnecessariamente pessimista que minimiza o maior valor (pior caso) da função objetivo nos conjuntos $\Xi \mathrm{e}$ $\Sigma$. Logo, o bom desempenho desta modelagem depende bastante de uma escolha criteriosa dos cenários que serão considerados no problema de min-max.

No que segue, será conveniente fazermos as seguintes definições para $u \geq \mathbf{0}, v \geq \mathbf{0}, \omega \in \Gamma, \varphi \in \Xi, \Lambda \in \Sigma$ :

$$
\Upsilon_{\varphi, \Lambda}(\omega, u)=\left\{\Lambda^{\frac{1}{2}}\left(u-\left(\mathcal{A}-\mathbf{1} \mu^{\prime}\right)\left(\omega-\omega_{b}\right)\right)\right\}
$$




$$
\Delta_{\varphi, \Lambda}(\omega, v)=\left\{\Lambda^{\frac{1}{2}}\left(v+\left(\mathcal{A}-\mathbf{1} \mu^{\prime}\right)\left(\omega-\omega_{b}\right)\right)\right\} .
$$

Definimos também

$$
\begin{aligned}
g_{\varphi, \Lambda}(\omega, u, v)= & -(1-\delta)\left(\omega-\omega_{b}\right)^{\prime}(\mu-\mathbf{1} r) \\
& +\delta \theta\left\|\Upsilon_{\varphi, \Lambda}(\omega, u)\right\|^{2} \\
& +\delta(1-\theta)\left\|\Delta_{\varphi, \Lambda}(\omega, v)\right\|^{2} .
\end{aligned}
$$

Proposição 3 Para cada $\omega \in \Gamma$ fixo,

$$
\begin{aligned}
& \sup _{\varphi \in \Xi, \Lambda \in \Sigma} f_{\varphi, \Lambda}(\omega)= \\
& \min _{u \geq \mathbf{0}, v \geq \mathbf{0}} \underset{k=1, \ldots, \kappa, \ell=1, \ldots, \nu}{\max } g_{\varphi(k), \Lambda(\ell)}(\omega, u, v) .
\end{aligned}
$$

\section{FORMULAÇÃO LMI}

Vamos introduzir agora uma formulação para o problema robusto de seleção de carteiras apresentado em (34) utilizando desigualdades matriciais lineares. Mostraremos, através de um teorema, que a formulação LMI para o problema (34) é:

Minimizar $z$

Sujeito a:

$$
\begin{aligned}
& {\left[\begin{array}{ccc}
\mathcal{S}_{0} & \mathcal{S}_{1} & \mathcal{S}_{2} \\
\mathcal{S}_{1}^{\prime} & I & 0 \\
\mathcal{S}_{2}^{\prime} & 0 & I
\end{array}\right] \geq 0} \\
& i=1, \ldots, \kappa, k=1, \ldots, \nu \\
& u \geq \mathbf{0}, v \geq \mathbf{0}, \omega \in \Gamma .
\end{aligned}
$$

com:

$$
\begin{aligned}
& \mathcal{S}_{0}=\left(z+(1-\delta)\left(\omega-\omega_{B}\right)^{\prime}(\mu(i)-\mathbf{1} r(i))\right) \\
& \mathcal{S}_{1}=(\delta \theta)^{\frac{1}{2}} \Upsilon_{\varphi(i), \Lambda(k)}(\omega, u)^{\prime} \\
& \mathcal{S}_{2}=(\delta(1-\theta))^{\frac{1}{2}} \Delta_{\varphi(i), \Lambda(k)}(\omega, v)^{\prime}
\end{aligned}
$$

e as variáveis são : $u, v, z, \omega$. Vale ressaltar que na equação (43) a desigualdade é para cada elemento dos vetores $T$ dimensional $u, v$, e $\mathbf{0}$ representa o vetor $T$-dimensional zero.

O resultado a seguir mostra que o problema de otimização utilizando desigualdades matriciais lineares apresentado acima (41 - 44) resolve o problema de otimização robusta de carteiras (34).

Teorema $1 \bar{\omega} \in \Gamma$ é uma solução ótima do problema inf sup posto em (34) se e somente se $\bar{u}, \bar{v}, \bar{z}, \bar{\omega}$ é solução ótima do problema LMI posto em (41) a (44). Mais ainda,

$$
\begin{aligned}
\bar{z}= & \max _{i=1, \ldots, \kappa, k=1, \ldots, \nu} \\
& \left\{-(1-\delta)\left(\bar{\omega}-\omega_{b}\right)^{\prime}(\mu(i)-\mathbf{1} r(i))\right. \\
+ & \delta(1-\theta)\left\|\Lambda(k)^{\frac{1}{2}}\left[\left(\mathcal{A}-\mathbf{1} \mu(i)^{\prime}\right)\left(\bar{\omega}-\omega_{b}\right)\right]_{+}\right\|^{2} \\
+ & \left.\delta \theta\left\|\Lambda(k)^{\frac{1}{2}}\left[\left(\mathcal{A}-\mathbf{1} \mu(i)^{\prime}\right)\left(\bar{\omega}-\omega_{b}\right)\right]_{-}\right\|^{2}\right\}
\end{aligned}
$$

$$
\begin{gathered}
\bar{u}=\left[\left(\mathcal{A}-\mathbf{1} \mu(\iota)^{\prime}\right)\left(\bar{\omega}-\omega_{b}\right)\right]_{+} \\
\bar{v}=-\left[\left(\mathcal{A}-\mathbf{1} \mu(\iota)^{\prime}\right)\left(\bar{\omega}-\omega_{b}\right)\right]_{-}
\end{gathered}
$$

e $\iota \in\{1, \ldots, \kappa\}, \xi \in\{1, \ldots, \nu\}$ atingem o máximo na equação (45).

Vale ressaltar que o problema de otimização utilizando desigualdades matriciais lineares colocado acima é equivalente ao seguinte problema de programação não-linear:

Minimizar $z$

Sujeito a:

$$
\begin{aligned}
& z+(1-\delta)\left(\omega-\omega_{b}\right)^{\prime}(\mu(i)-\mathbf{1} r(i))+ \\
& \delta \theta(u-\tau(i))^{\prime} \Lambda(k)(u-\tau(i))+ \\
& \delta(1-\theta)(v+\tau(i))^{\prime} \Lambda(k)(v+\tau(i)) \geq 0 \\
& i=1, \ldots, \kappa, k=1, \ldots, \nu \\
& u \geq \mathbf{0}, v \geq \mathbf{0} \\
& \omega \in \Gamma
\end{aligned}
$$

no qual $\tau(i)=\left(\mathcal{A}-\mathbf{1} \mu(i)^{\prime}\right)\left(\omega-\omega_{b}\right)$.

Assim sendo, podemos utilizar também métodos para problemas de programação não-linear para resolver o problema robusto de carteiras. A vantagem de utilizar desigualdades matriciais lineares é que este tipo de formulação é tratável tanto do ponto de vista teórico como numérico, e existem hoje códigos bastante eficientes para a obtenção de soluções.

Para o caso no qual temos $\theta=1$, o problema de otimização (41)-(44) pode ser simplificado para:

Minimizar $z$

Sujeito a:

$$
\begin{aligned}
& {\left[\begin{array}{cc}
\mathcal{S}_{0} & \mathcal{S}_{1} \\
\mathcal{S}_{1}^{\prime} & I
\end{array}\right] \geq 0} \\
& i=1, \ldots, \kappa, k=1, \ldots, \nu \\
& u \geq \mathbf{0}, \omega \in \Gamma,
\end{aligned}
$$

e de forma análoga para $\theta=0$. Para o caso de termos $\theta=\frac{1}{2}$, o problema de otimização (41)-(44) pode ser simplificado a

\section{Minimizar $z$}

\section{Sujeito a:}

$$
\begin{gathered}
{\left[\begin{array}{cc}
\mathcal{S}_{0} & \delta^{\frac{1}{2}}\left(\omega-\omega_{B}\right)^{\prime} \Omega(i, k) \\
\delta^{\frac{1}{2}} \Omega(i, k)\left(\omega-\omega_{B}\right) & \Omega(i, k)
\end{array}\right] \geq 0} \\
i=1, \ldots, \kappa, k=1, \ldots, \nu, \omega \in \Gamma \\
\text { sendo } \Omega(i, k)=\left(\mathcal{A}-\mathbf{1} \mu(i)^{\prime}\right)^{\prime} \Lambda(k)\left(\mathcal{A}-\mathbf{1} \mu(i)^{\prime}\right) .
\end{gathered}
$$

\section{EXEMPLOS NUMÉRICOS}

Nesta seção apresentaremos alguns exemplos numéricos com base na formulação LMI apresentada acima. 
Dada a diversidade de ativos e indicadores existentes no mercado brasileiro, decidimos estabelecer convenções para a realização das simulações. Em primeiro lugar adotamos o Índice da Bolsa de Valores de São Paulo, o IBOVESPA, como nosso benchmark e, assim sendo, selecionamos um conjunto de ações negociadas nesta Bolsa de Valores para o cálculo da carteira robusta.

O IBOVESPA é o valor, em moeda corrente, de uma carteira teórica de ações e um dos mais importantes indicadores do desempenho médio das cotações do mercado de ações brasileiro. O seu valor é apurado diariamente como sendo o somatório dos pesos (quantidade teórica da ação multiplicada pelo último preço da mesma) das ações integrantes de sua carteira teórica. E para determinar quais os ativos irão compor esta carteira teórica, a Bolsa de Valores de São Paulo utiliza uma metodologia onde os aspectos mais importantes são a liquidez e o índice de negociabilidade, que é calculado com base no número de negócios e volume financeiro, das ações.

Na prática, estas 2 informações são muito importantes para o investidor no momento da escolha dos ativos que irão compor sua carteira. Mais ainda, como o intuito do nosso modelo é obter uma carteira que melhor acompanhe a variação do benchmark, que é o IBOVESPA, decidimos então utilizar as 37 ações que foram mais representativas na composição da carteira téorica deste índice ao longo de 2001, de forma que a soma das participações individuais de cada uma delas tenham representado, durante o período, no mínimo $85 \%$ da participação total.

Assim sendo, calculamos a carteira ótima semanalmente para o primeiro semestre de 2001, e utilizamos os últimos 63 dados disponíveis para construir a matriz de retornos $\mathcal{A}$. Assumimos ainda que a riqueza inicial disponível para investimento era 100 unidades monetárias.

Para as simulações numéricas utilizamos os seguintes valores para os parâmetros:

1. números de LMIs:
(a) $\kappa=1, \nu=1$ (uma LMI);
(b) $\kappa=2, \nu=2$ (quatro LMIs);

2. $\delta$ :
(a) $\delta 1=0,94$;
(b) $\delta 2=0,97$;
(c) $\delta 3=0,99$;

3. $\theta=0,75$;
4. matriz $\Lambda$ :
(a) $\Lambda(1)=\left[\begin{array}{ccccc}\frac{1}{63} & 0 & \ldots & 0 & 0 \\ 0 & \frac{1}{63} & \ldots & 0 & 0 \\ \vdots & \vdots & \ddots & \vdots & \vdots \\ 0 & 0 & \ldots & \frac{1}{63} & 0 \\ 0 & 0 & \ldots & 0 & \frac{1}{63}\end{array}\right]$;
(b) $\Lambda(2)=\frac{0,1}{c}\left[\begin{array}{cccc}0,9^{62} & 0 & \ldots & 0 \\ 0 & 0,9^{61} & \ldots & 0 \\ \vdots & \vdots & \ddots & \vdots \\ 0 & 0 & \ldots & 1\end{array}\right]$;

5. Amostra de Dados: 63 dias úteis (aprox. 3 meses);

sendo $c$ um fator de normalização. Em ambos os casos calculamos o vetor $\mu$ de acordo com a média móvel dos últimos 63 dias, e utilizamos os pesos dos ativos selecionados na composição do Ibovespa para obter o vetor $\omega_{B}$. Consideramos o ativo livre de risco $r_{k}$ como o CDI-Cetip.

Geramos 3 conjuntos diferentes de carteiras. Para a Simulação 1 temos:

- números de LMIs = 1

- $\delta=\delta 1$

- matrizes $\Lambda=\Lambda(1)$ (cenário 1 )

Para Simulação 2 consideramos:

- números de LMIs = 4

- $\delta=\delta 2$

- matrizes $\Lambda=\Lambda(1)$ (cenário 1) e $\Lambda(2)$ (cenário 2)

E para Simulação 3:

- números de LMIs = 4

- $\delta=\delta 3$

- matrizes $\Lambda=\Lambda(1)$ (cenário 1) e $\Lambda(2)$ (cenário 2)

Vale ressaltar que no caso da Simulação 1 estamos utilizando a formulação original proposta por Markowitz em (Markowitz, 1952). Desta forma podemos comparar os resultados obtidos com a metodologia aqui proposta com outras, bem como verificar o impacto dos parâmetros nos modelos. 


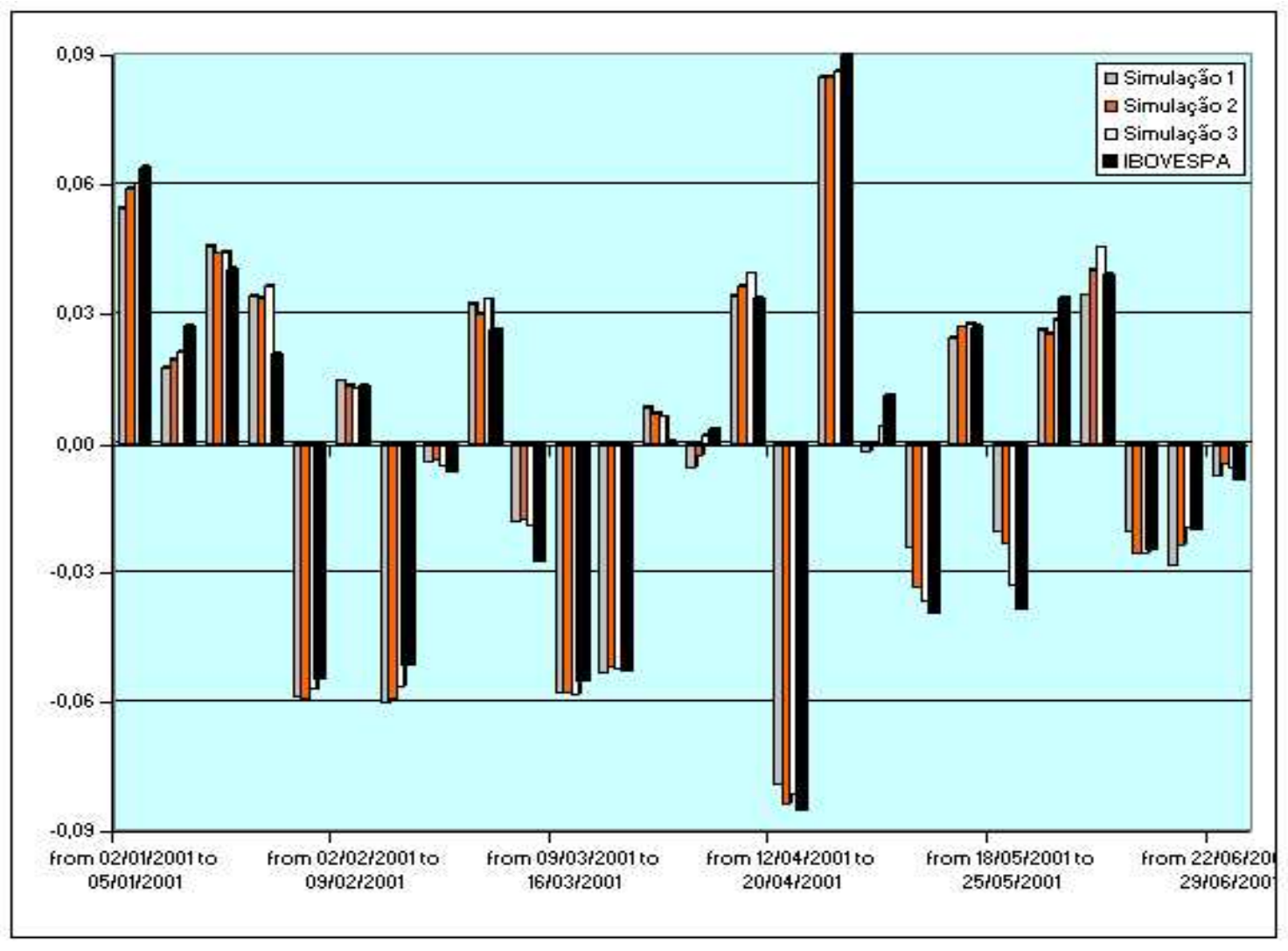

Figura 1: Retornos Semanais do IBOVESPA e das Simulações 1, 2 e 3

Os resultados foram obtidos utilizando um solver chamado LMISol desenvolvido por Oliveira, de Faria e Geromel da Faculdade de Engenharia Elétrica e de Computação da Universidade Estadual de Campinas, e disponível em (Oliveira et al., 1994).

Vale colocar que não faz parte do escopo deste trabalho um estudo mais profundo sobre a questão da geração dos cenários, portanto utilizamos apenas pesos diferentes (matrizes $\Lambda$ ) para o cálculo das estimativas de média e semi-variâncias como forma de diferenciá-los.

No primeiro gráfico temos a variação semanal das 4 carteiras (Simulação 1, Simulação 2, Simulação 3 e o Ibovespa). No segundo temos o comportamento das carteiras ao longo do tempo, dado que o investimento inicial foi 100

Com base nos gráficos apresentados, principalmente considerando o gráfico de rentabilidade acumulada, podemos notar que as 3 simulações realizadas apresentaram resultados satisfatórios, ou seja, acompanharam a variação do benchmark e conseguiram um rendimento superior ao IBOVESPA no período considerado.
Neste contexto podemos fazer 2 análises: comparar modelos distintos (Simulação 1 versus Simulações 2 e 3), bem como analisar o impacto da parametrização nos resultados do modelo robusto (Simulação 2 versus Simulação 3).

Com relação à comparação de metodologias distintas, podemos observar que, apesar do modelo robusto ser uma análise de pior caso, uma escolha conveniente do conjunto de parâmetros pode produzir resultados melhores do que os obtidos utilizando a metodologia clássica de Markowitz onde consideramos apenas um cenário de risco e retorno. Por exemplo, quando comparamos as Simulações 1 e 2 e Simulações 1 e 3, dado que definimos para as Simulações 2 e 32 cenários de risco e retorno, sendo um deles o mesmo utilizado no modelo de Markowitz (Simulação 1), era esperado que o modelo robusto fornecesse um resultado de pior caso, ou seja, apresentasse uma rentabilidade inferior. No entanto, principalmente quando comparamos com a Simulação 3, podemos observar justamente o inverso, ou seja, o modelo robusto fornece um resultado melhor do que o modelo clássico.

Quando comparamos 2 simulações utilizando o mesmo modelo (Simulação 2 versus Simulação 3), uma vez que mantivemos os mesmos cenários mas obtivemos respostas dife- 


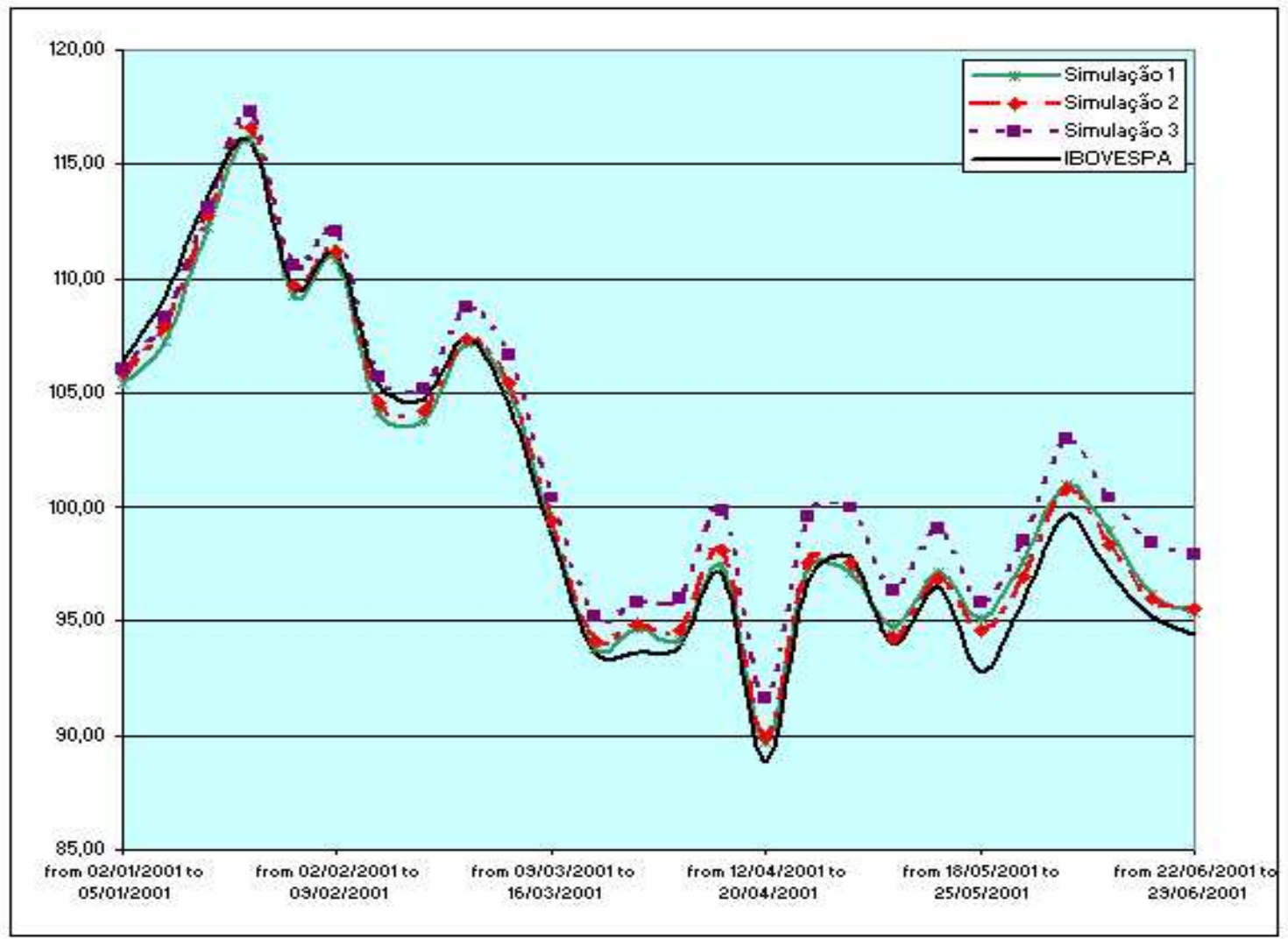

Figura 2: Retorno Acumulado do IBOVESPA e das Simulações 1, 2 e 3

rentes do modelo, podemos dizer que a escolha dos parâmetros $\theta$ e $\delta$ são tão importantes quanto a determinação dos cenários econômicos. Ou seja, não adianta termos uma boa projeção de risco e retorno dos ativos se a resposta do modelo não atende às necessidades do investidor. Portanto, mais do que a coerência e correção matemática do modelo proposto, a sua utilização em condições reais de mercado depende muito do investidor/usuário da ferramenta.

Em termos práticos, este é um aspecto bastante interessante pois sempre que modificamos nossas estimativas de risco e retorno ( $\theta$ e cenários) estamos, de alguma maneira, tentando prever o comportamento futuro do mercado financeiro, o que não é uma tarefa trivial! No entanto, uma vez que nossas "previsões" sejam coerentes, é preciso estabelecer os objetivos do investidor, ou seja, dado um conjunto de alternativas de investimento (quais ativos bem como suas expectativas de retorno e risco) qual a decisão será tomada

Mais informações sobre simulações numéricas do modelo proposto podem ser encontradas em (Rodrigo, 2002).

\section{CONCLUSÕES}

Neste artigo consideramos o problema de seleção ótima de ativos usando semivariâncias como uma medida para a função risco do erro de rastreamento. Assumimos que os retornos esperados dos ativos e do ativo livre de risco, bem como os pesos para o cálculo das semivariâncias, pertencem a um politopo convexo definido por alguns elementos conhecidos que formam os vértices do politopo. $\mathrm{O}$ valor da função objetivo é definido como uma combinação convexa da função risco menos o retorno esperado do erro de rastreamento. Mostramos ainda que o problema de encontrar a carteira com o mínimo pior caso da função objetivo é equivalente a resolver um problema de otimização com desigualdades matriciais lineares, de forma a possibilitar o uso de ferramentas poderosas disponíveis para resolução desta classe de problemas. Apresentamos simulações numéricas com ações negociadas na Bovespa, usando dados do primeiro semestre de 2001. Os resultados computacionais obtidos sugerem que o uso deste tipo de formulação pode ser bastante útil em problemas de otimização robusta de carteiras. 


\section{APÊNDICE: DEMONSTRAÇÕES}

Neste apêndice apresentaremos as demonstrações do Corolário 1, Teorema 1 e Proposições 2, 3.

\section{Corolário 1}

Prova. Da Proposição 1 segue que para cada $i$

$$
\left[\begin{array}{cc}
x(i)^{\prime} Q x(i) & x(i)^{\prime} Q^{1 / 2} \\
Q^{1 / 2} x(i) & I
\end{array}\right] \geq 0
$$

e portanto

$$
\begin{gathered}
\sum_{i=1}^{k} \alpha(i)\left[\begin{array}{cc}
x(i)^{\prime} Q x(i) & x(i)^{\prime} Q^{1 / 2} \\
Q^{1 / 2} x(i) & I
\end{array}\right]= \\
=\left[\begin{array}{cc}
\sum_{i=1}^{k} \alpha(i) x(i)^{\prime} Q x(i) & \sum_{i=1}^{k} \alpha(i) x(i)^{\prime} Q^{1 / 2} \\
\sum_{i=1}^{k} \alpha(i) Q^{1 / 2} x(i) & I
\end{array}\right] \geq 0 .
\end{gathered}
$$

O resultado desejado segue da aplicação da Proposição 1 na desigualdade acima.

\section{Proposição 2}

Prova. Como $x=[x]_{+}+[x]_{-}$segue que

$$
\begin{aligned}
\|\Upsilon(x, u)\|^{2}= & \left\|\Lambda^{\frac{1}{2}}\left(\left(u-[x]_{+}\right)-[x]_{-}\right)\right\|^{2} \\
= & \left\|\Lambda^{\frac{1}{2}}\left(u-[x]_{+}\right)\right\|^{2}+\left\|\Lambda^{\frac{1}{2}}[x]_{-}\right\|^{2} \\
& -2\left(u-[x]_{+}\right)^{\prime} \Lambda[x]_{-} .
\end{aligned}
$$

Mas de (7)

$$
\begin{aligned}
-\left(u-[x]_{+}\right)^{\prime} \Lambda[x]_{-} & =-u^{\prime} \Lambda[x]_{-}+[x]_{+}^{\prime} \Lambda[x]_{-} \\
& =-u^{\prime} \Lambda[x]_{-} \geq 0
\end{aligned}
$$

como $u \geq \mathbf{0},-[x]_{-} \geq \mathbf{0}$ e $\Lambda$ é uma matriz diagonal com componentes positivos. Portanto de (48) para qualquer $u \geq \mathbf{0}$

$$
\|\Upsilon(x, u)\|^{2} \geq\left\|\Lambda^{\frac{1}{2}}\left(u-[x]_{+}\right)\right\|^{2}+\left\|\Lambda^{\frac{1}{2}}[x]_{-}\right\|^{2}
$$

e é possível ver que o mínimo de (49) é atingido em $u=$ $[x]_{+}$, com valor $\left\|\Lambda^{\frac{1}{2}}[x]_{-}\right\|^{2}$. Analogamente o mínimo de $\Delta(y, v)$ sobre $v \in \mathbb{R}^{n}, v \geq \mathbf{0}$ é atingido em $v=-[y]_{-}$com valor $\left\|\Lambda^{\frac{1}{2}}[y]_{+}\right\|^{2}$. Isto completa a prova.

\section{Proposição 3}

Prova. Para quaisquer $u \geq \mathbf{0}, v \geq \mathbf{0}, \varphi \in \Xi, \Lambda \in \Sigma$, com

$$
\begin{gathered}
\varphi=\sum_{i=1}^{\kappa} \alpha(i) \varphi(i), \\
\sum_{i=1}^{\kappa} \alpha(i)=1, \alpha(i) \geq 0,
\end{gathered}
$$

$$
\Lambda=\sum_{k=1}^{\nu} \beta(k) \Lambda(k),
$$

e

$$
\sum_{k=1}^{\nu} \beta(k)=1, \beta(k) \geq 0,
$$

temos da Proposição 2 que

$$
g_{\varphi, \Lambda}(\omega, u, v) \geq f_{\varphi, \Lambda}(\omega)
$$

e o ponto de mínimo no lado esquerdo de (50) para $u \geq$ $0, v \geq 0$ é $f_{\varphi, \Lambda}(\omega)$, atingido em

$$
\begin{gathered}
u=\left[\left(\mathcal{A}-\mathbf{1} \mu^{\prime}\right)\left(\omega-\omega_{B}\right)\right]_{+}, \\
v=-\left[\left(\mathcal{A}-\mathbf{1} \mu^{\prime}\right)\left(\omega-\omega_{B}\right)\right]_{-} .
\end{gathered}
$$

Vamos definir então

$$
\chi_{u}=\left(u-\left(\mathcal{A}-\mathbf{1} \mu(i)^{\prime}\right)\left(\omega-\omega_{B}\right)\right),
$$

e

$$
\chi_{v}=\left(v+\left(\mathcal{A}-\mathbf{1} \mu(i)^{\prime}\right)\left(\omega-\omega_{B}\right)\right) .
$$

Do Corolário 1 temos

$$
\begin{gathered}
\left\|\Upsilon_{\varphi, \Lambda}(\omega, u)\right\|^{2}= \\
=\left\|\Lambda^{\frac{1}{2}}\left(u-\left(\mathcal{A}-\mathbf{1} \sum_{i=1}^{\kappa} \alpha(i) \mu(i)^{\prime}\right)\left(\omega-\omega_{B}\right)\right)\right\|^{2} \\
=\left\|\Lambda^{\frac{1}{2}}\left(\sum_{i=1}^{\kappa} \alpha(i) \chi_{u}\right)\right\|^{2} \\
\leq \sum_{i=1}^{\kappa} \alpha(i)\left\|\Lambda^{\frac{1}{2}} \chi_{u}\right\|^{2}
\end{gathered}
$$

e como

$$
\begin{gathered}
\left.\| \Upsilon_{\varphi(i), \Lambda}(\omega, u)\right) \|^{2}= \\
=\left\|\Lambda^{\frac{1}{2}}\left(u-\left(\mathcal{A}-\mathbf{1} \mu(i)^{\prime}\right)\left(\omega-\omega_{B}\right)\right)\right\|^{2} \\
=\chi_{u}^{\prime} \sum_{k=1}^{\nu} \beta(k) \Lambda(k) \chi_{u} \\
=\sum_{k=1}^{\nu} \beta(k) \chi_{u}^{\prime} \Lambda(k) \chi_{u}
\end{gathered}
$$

temos que

$$
\begin{aligned}
\left\|\Upsilon_{\varphi, \Lambda}(\omega, u)\right\|^{2} & \leq \sum_{i=1}^{\kappa} \sum_{k=1}^{\nu} \beta(k) \alpha(i)\left\|\Lambda(k)^{\frac{1}{2}} \chi_{u}\right\|^{2} \\
& =\sum_{i=1}^{\kappa} \sum_{k=1}^{\nu} \beta(k) \alpha(i)\left\|\Upsilon_{\varphi(i), \Lambda(k)}(\omega, u)\right\|^{2}
\end{aligned}
$$

Analogamente

$$
\begin{aligned}
\|\left.\Delta_{\varphi, \Lambda}(\omega, v)\right|^{2} & \leq \sum_{i=1}^{\kappa} \sum_{k=1}^{\nu} \beta(k) \alpha(i)\left\|\Lambda(k)^{\frac{1}{2}} \chi_{v}\right\|^{2} \\
& =\sum_{i=1}^{\kappa} \sum_{k=1}^{\nu} \beta(k) \alpha(i)\left\|\Delta_{\varphi(i), \Lambda(k)}(\omega, u)\right\|^{2} .
\end{aligned}
$$


Como

$$
\begin{gathered}
\left(\omega-\omega_{B}\right)^{\prime}(\mu-\mathbf{1} r)= \\
\sum_{i=1}^{\kappa} \alpha(i)\left(\omega-\omega_{B}\right)^{\prime}(\mu(i)-\mathbf{1} r(i))
\end{gathered}
$$

podemos concluir que

$$
g_{\varphi, \Lambda}(\omega, u, v) \leq \sum_{i=1}^{\kappa} \sum_{k=1}^{\nu} \beta(k) \alpha(i) g_{\varphi(i), \Lambda(k)}(\omega, u, v) .
$$

Desta forma,

$$
\sup _{\varphi \in \Xi, \Lambda \in \Sigma} g_{\varphi, \Lambda}(\omega, u, v)=\max _{i, k} g_{\varphi(i), \Lambda(k)}(\omega, u, v)
$$

e o máximo é alcançado para algum $i \in\{1, \ldots, \kappa\}, k \in$ $\{1, \ldots, \nu\}$. Tomando o mínimo sobre $u \geq \mathbf{0}, v \geq \mathbf{0}$ obtemos a partir de (50) o resultado desejado.

\section{Teorema 1}

Prova. Vamos fixar $\omega \in \Gamma$. Conforme a Proposição 1 a desigualdade (42) é equivalente a

$$
z \geq g_{\varphi(i), \Lambda(k)}(\omega, u, v)
$$

e deve ser satisfeita para todo $i=1, \ldots, \kappa, k=1, \ldots, \nu$. Portanto de (51) temos

$$
z \geq \max _{i=1, \ldots, \kappa, k=1, \ldots, \nu} g_{\varphi(i), \Lambda(k)}(\omega, u, v) .
$$

Como queremos minimizar $z$, a melhor escolha é tomar o mínimo sobre $u \geq \mathbf{0}, v \geq \mathbf{0}$. Segue então da Proposição 3 que

$$
\begin{aligned}
z & \geq \min _{u \geq \mathbf{0}, v \geq \mathbf{0}} \max _{k=1, \ldots, \kappa, \ell=1, \ldots, \nu} g_{\varphi(k), \Lambda(\ell)}(\omega, u, v) \\
& =\sup _{\varphi \in \Xi, \Lambda \in \Sigma} f_{\varphi, \Lambda}(\omega) .
\end{aligned}
$$

e o mínimo é obtido para $u$ e $v$ como nas equações (45), (46), (47). Então de (52), minimizar (41) sujeito às restrições (42) a (44) é equivalente a resolver (34).

\section{REFERÊNCIAS}

Alexander, A. J. e Sharpe, W. F. (1989). Fundamentals of Investment. Prentice-Hall, New Jersey.

Black, F. e Litterman, R. (1991). Asset allocation: Combining investor views with market equilibrium. Journal of Fixed Income, 1, 7-18.

Bollerslev, T. (1986). Generalized autoregressive conditional heteroskedasticity. Journal of Econometrics, 31, 307327.

Bollerslev, T., Chou, R. Y. e Kroner, K. F. (1992). ARCH Modelling in Finance: A review of theory and empirical evidences. Journal of Econometrics, 52, 5-59.
Boyd, S., Ghaoui, L. E., Feron, E. e Balakrishnan, V. (1994). Linear Matrix Inequalities and Control Theory. SIAM, Philadelphia.

Campbell, J. Y., Lo, A. W. e MacKinlay, A. C. (1997). The Econometrics of Financial Markets. Princeton University Press.

Costa, O. L. V., do Val, J. B. R. e Geromel, J. C. (1997). A convex programming approach to $\mathrm{H}_{2}$-control of markovian jump linear systems. International Journal of Control, 66, 557-579.

Costa, O. L. V. e Nabholz, R. B. (2002). Robust mean-semivariance portfolio optimization, in Kontoghiorghes, E. J., Rustem, B., Siokos, S., editors Computational Methods in Decision-Making, Economics and Finance, Kluwer Academic Publishers.

Costa, O. L. V. e Paiva, A. C. (2002). Robust portfolio selection using linear matrix inequalities. Journal of Economic Dynamics and Control 26: 889-909.

Dembo, R. S. (1991). Scenario Optimization. Annals of Operations Research, 30, 63-80.

Duarte Jr., A. M. (1999). Fast computation of efficient portfolios, The Journal of Risk, 4: 71-94.

Elton, E. J. e Gruber, M. J. (1995). Modern Portfolio Theory and Investment Analysis. Wiley, New York.

Enders, W. (1995). Applied Econometric Time Series. John Wiley, New York.

Engel, J. e Gizycki, M. (1999). Value at Risk : On the stability and forecasting of the variance-covariance matrix. Research Discussion Paper 1999-04, Reserve Bank of Australia, Australia.

Engle, R. F. (1992). Autoregressive conditional heteroskedasticity with estimates of the variance of United Kingdon inflation. Econometrica, 50, 987-1007.

Geromel, J. C., Peres, P. L. D. e Bernussou, J. (1991). On a convex parameter space method for linear control design of uncertain systems. SIAM J. on Control and Optimization, 29, 381-402.

Ghaoui, L. E. e Niculescu, S. I. (2000). Advances in Linear Matrix Inequality Methods in Control. SIAM, Philadelphia.

Hanza, F. e Janssen, J. (1995). Portfolio optimization model using asymmetric risk function. Proc.5th AFIR, Brussels, Belgium, Vol III bis, pp. 3-32. 
Hanza, F. e Janssen, J. (1998). The mean-semivariances approach to realistic portfolio optimization subject to transactions costs. Applied Stochastics Models and Data Analysis, 14, 275-283.

Howe, M. A. e Rustem, B. (1997). A robust hedging algorithm. Journal of Economic Dynamics and Control, 21: 1065-1092.

Howe, M. A., Rustem, B. e Selby, M. J. P. (1996). Multiperiod minimax hedging strategies. European Journal of Operational Research, 93: 185-204.

Jorion, P. (1992). Portfolio optimization in practice. Financial Analysts Journal, (January-February), 1: 68-74.

Luenberger, D. G. (1997). Investment Science. Oxford University Press.

Mills, T. C. (1993). The Econometric Modelling of Financial Time Series. Cambridge University Press.

Markowitz, H. M. (1952). Portfolio Selection. Journal of Finance, 7, 77-91.

Markowitz, H. M. (1959). Portfolio Selection: Efficient Diversification of Investments. John Wiley, New York.

Markowitz, H. M. (1987). Mean-Variance Analysis in PorTfolio Choice and Capital Markets. Basil Blackwell.

Nabholz, R. B. (2002). Otimização Robusta de Carteiras utilizando Desigualdades Matriciais Lineares. Dissertação de Mestrado, Escola Politécnica, USP, São Paulo, Brasil.

Oliveira, M. C., Faria, D. P. e Geromel, J. C. (1994). Lmisol user's guide, Available at www.dt.fee.unicamp.br/ carvalho/\#soft.

Roll, R. (1992). A mean/variance analysis of tracking error. The Journal of Portfolio Management, 18, 13-22.

Rudolf, M., Wolter, H. J. e Zimmermann, H. (1999). A linear model for tracking error minimization. The Journal of Banking and Finance, 23, 85-103.

Rustem, B., Becker, R. G. e Marty, W. (1995). Robust minmax portfolio strategies for rival forecast and risk scenarios. Journal of Economic Dynamics and Control, 24, 1591-1621.

Saberi, A., Sannuti, P. e Chen, M. B. (1995). $\mathrm{H}_{2}$-Optimal Control. Prentice Hall.

Steinbach, M. C. (2001). Markowitz Revisited: MeanVariance Models in Financial Portfolio Analysis. SIAM Review, 43, 31-85.

Zenios, S. A. (1993). Financial Optimization. Cambridge University Press. 